\title{
Identifying Patients with Myasthenia for Epidemiological Research by Linkage of Automated Registers
}

\author{
Emil Greve Pedersen ${ }^{a}$ Jesper Hallas ${ }^{b}$ Klaus Hansen ${ }^{d}$ Poul Erik Hyldgaard Jensen ${ }^{e}$ \\ David Gaist ${ }^{\text {a, c }}$ \\ ${ }^{a}$ Department of Neurology, Odense University Hospital, Odense, ${ }^{b}$ Institute of Public Health, Clinical Pharmacology \\ Unit, and ' Institute of Clinical Research, University of Southern Denmark, and ${ }^{\mathrm{d}}$ Department of Neurology and \\ e Neuroimmunology Laboratory, DMSC, Department of Neurology, Rigshospitalet, Copenhagen University \\ Hospital, Copenhagen, Denmark
}

\section{Key Words}

Myasthenia $\cdot$ Neuromuscular diseases $\cdot$ Neurological disorders $\cdot$ Epidemiology $\cdot$ Research methods

\begin{abstract}
Background: We validated a new method of identifying patients with incident myasthenia in automated Danish registers for the purpose of conducting epidemiological studies of the disorder. Methods: For residents of a Danish county (population 484,862) in 1993-2008, we identified any hospital contacts coded for myasthenia in a nationwide patient register and any prescriptions for pyridostigmine in the county prescription register. Results from an acetylcholine receptor antibody register were linked to the data. We verified the diagnosis by a review of medical records. Results: Subjects identified in the Patient Register $(n=83)$ were comparable with individuals found in the Prescription Register $(n=89)$ with regard to age and gender, but were more often seropositive ( 83.1 vs. $74.2 \%)$. Seropositivity increased to $91.6 \%$ by restricting the data to individuals recorded in both Patient and Prescription Registers $(n=71)$. We found that for subjects identified in both Patient and Prescription Registers
\end{abstract}

the positive predictive value of the register diagnosis was 92.9\% (95\% confidence interval, $\mathrm{Cl}, 84.3-97.7)$, the false-positive rate was low $(2.8 \%)$, and the sensitivity was acceptable (81.2\%; 95\% Cl 71.2-88.8). Conclusions: Our data indicate that this novel approach of combining diagnosis register and prescription register information provides a feasible and valid method to trace incident myasthenia patients for population-based epidemiological studies.

Copyright $\odot 2011$ S. Karger AG, Basel

\section{Introduction}

Acquired autoimmune myasthenia gravis (myasthenia) is a disorder of the neuromuscular junction causing muscle weakness and fatiguability [1]. The rarity of myasthenia combined with the need of expertise to recognize and correctly diagnose the disorder makes largescale population-based studies of myasthenia with ad hoc collection of data on the disorder arduous and timeconsuming. Studies based on data from automated registers that enable timely identification of large samples of cases are therefore increasingly being utilized in my-

\section{KARGER}

Fax +41 613061234 E-Mail karger@karger.ch www.karger.com

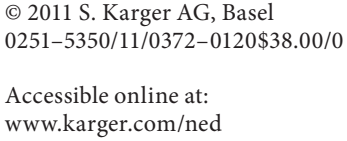

David Gaist
Department of Neurology, Odense University Hospital
Sdr Boulevard 29
DK-5000 Odense C (Denmark)
Tel. +45 6541 2498, E-Mail davidgaist.neuro@ gmail.com 
asthenia epidemiological research [2-5]. We wished to use a nationwide Danish patient register for this purpose. However, in a previous Danish study that used hospital patient registers as a single source for case identification, the diagnosis could not be verified by medical record review in roughly $27 \%$ of cases registered under myasthenia codes [6]. We speculated whether combining information from patient registers with information from other registers would improve validity. This method could be implemented in a nationwide setting with relative ease. This would path the way for epidemiological studies of myasthenia that could utilize the large number of population-based registers available in Denmark [7].

Pyridostigmine, an acetylcholine esterase inhibitor, is used in practically all patients with clinically active myasthenia as the first line of therapy in Denmark. We assessed the validity of a diagnosis code for myasthenia in a Danish nationwide patient register and tested whether combining data from this source with prescription register information on pyridostigmine use enhanced the validity of the diagnosis code.

\section{Material and Methods}

The study focused on a geographically well-defined area of Denmark, the former County of Funen (population 484,862), henceforth referred to as the County. The department of Neurology at Odense University Hospital (OUH) serves as the referral centre for patients with neurological disorders in the area, and the entire County is covered by a prescription register.

Through registers we identified individuals with a myasthenia diagnosis code, a presented prescription of pyridostigmine, or an acetylcholine receptor antibody (AChRab) test result.

We ascertained the validity of the diagnosis both indirectly, by linkage to Antibody Register data, and directly, by appraisal of medical records of potential cases of myasthenia. To increase generalizability with regard to the Patient Register myasthenia diagnosis, we also validated samples from other hospitals in Denmark.

Each resident of Denmark has a permanent unique civil registration number that is provided at birth or immigration to the country. This enabled simple and correct linkage of 4 registers:

(1) The Danish National Hospital Register (Patient Register) includes information on discharges (since 1977) and on outpatient visits (since 1995) from hospitals in Denmark (population 5.5 million) [8]. Patient-specific data include contact dates, department and hospital code, and diagnoses coded according to the Danish version of the International Classification of Diseases, 8th revision (ICD-8) from 1977 to 1993 and ICD-10 from 1994 onwards.

(2) The Odense University Pharmacoepidemiological Database (Prescription Register) has offered complete coverage of the County since November 1992 [9]. For each prescription, the date it was presented and a full account of the dispensed product including the anatomical therapeutic chemical code [10] are recorded.

(3) The Antibody Register was created by us by merging data on AChRab results from the Neuroimmunology Laboratory at Rigshospitalet $(\mathrm{RH})$, Copenhagen University Hospital, and the laboratory at OUH. Both laboratories provide in-service, as well as analyses of blood samples from other hospitals, general practitioners and privately practising neurologists. We classified individuals as 'positive' if a positive result was registered in either register at any time. The remaining individuals were classified as 'negative'. Within each category the earliest date of blood sampling was used as the date of antibody status.

The measurements of AChRab performed at RH (1977-2003) were analysed by an in-house radioimmunoassay method [11]. The RH laboratory at Copenhagen University Hospital has since 2004 used a commercial kit for ${ }^{125}$ I-radioimmunoassay quantification of AChRabs (DLD Diagnostika GmbH, Hamburg, Germany) [12]. The OUH laboratory used another commercial kit for the ${ }^{125}$ I-radioimmunoassay of AChRab (IBL-international, Hamburg, Germany) throughout the period 2000-2008.

(4) The Danish Civil Registration System has since 1968 recorded all persons residing in Denmark with their civil registration number, a 10-digit unique and permanent identifier [13]. The birth date, gender and data on residency are recorded in the register. Aggregated data on the background population's age and gender distribution were retrieved from Statistics Denmark (www.dst.dk).

\section{Identification and Validation of Potential Incident Cases of} Myasthenia in the County

We identified all discharges (from 1977 to 2008) and all outpatient visits (from 1995 to 2008) at Danish hospitals with a recorded primary diagnosis code for myasthenia (ICD-8: 733.9, ICD-10: G70.0) in the Patient Register and classified hospital contacts according to department type (neurology or non-neurology). The date of the first contact ever with a myasthenia code was identified for each person in the Patient Register sample. From the Prescription Register we retrieved all data available in the register from 1990 to 2008 on prescriptions for pyridostigmine (anatomical therapeutic chemical code: N07AA02) and included subjects that presented at least 2 prescriptions. Data from the Antibody Register were classified with regard to seropositivity as previously described. For individuals identified in any of the 3 registers we established residency status through linkage with the Civil Registration System. Individuals thus identified were classified according to whether data were registered in the Patient Register or the Prescription Register. Subjects were further subdivided according to whether they were recorded in both the Patient and the Prescription register, or exclusively in one of the registers (Patient only, Prescription only). Since we regarded the Antibody Register data as an indirect validation source of the other two registers, we did not create a separate group for subjects with positive antibodies only.

We wished to identify incident cases of myasthenia in the years 1993-2008. For each subject in the initial sample, we identified the index date, defined as the first registered date in the Patient, Prescription or Antibody Register, whichever came first. We included subjects if their index date was between January 1 , 


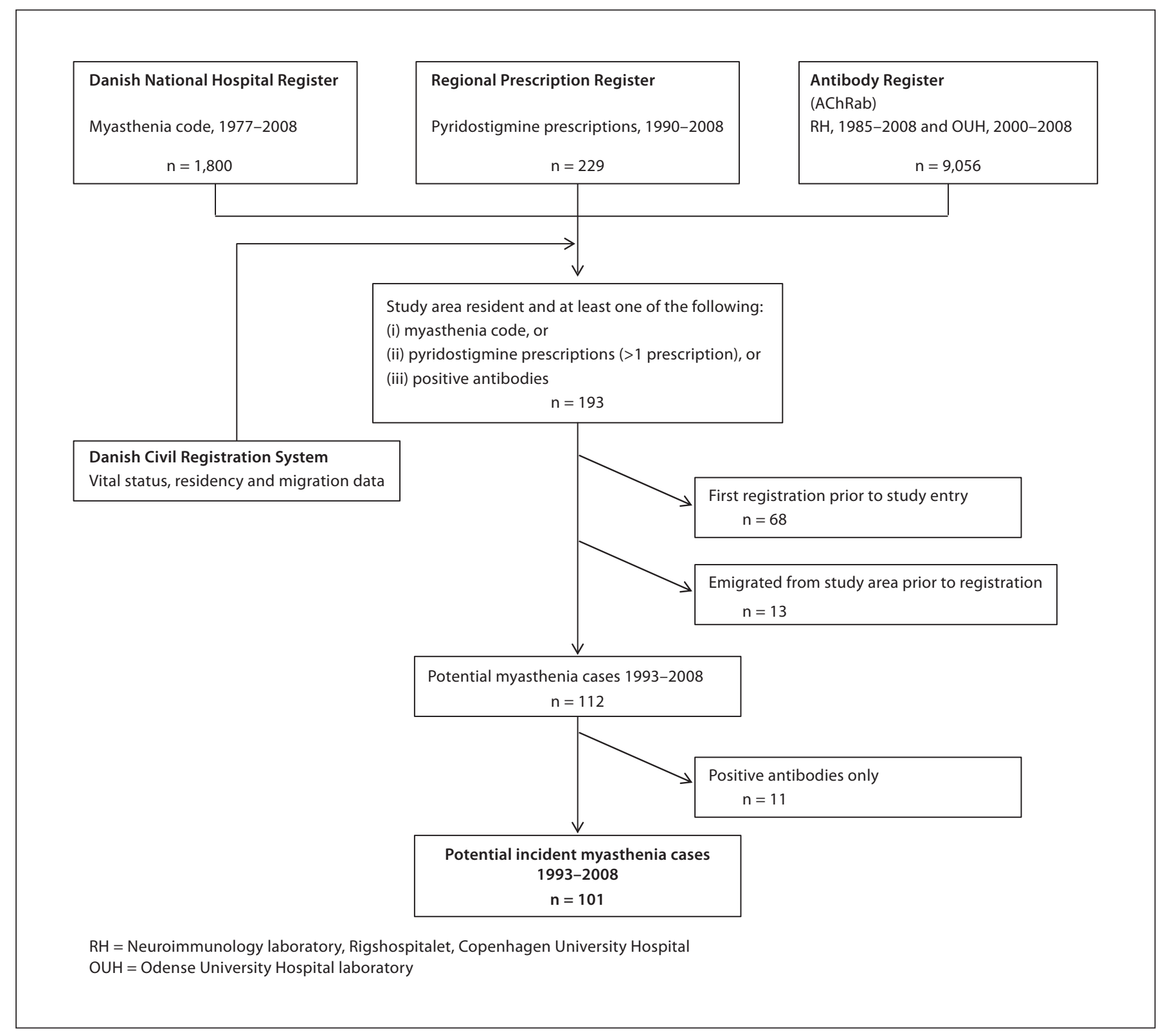

Fig. 1. Flow diagram of register-based identification of potential incident cases of myasthenia in a Danish county, $1993-2008$.

1993, and December 31, 2008. We excluded a small number of potential cases under 16 years of age $(n=4)$, since prescriptions issued to children in this age group were registered under one of their parent's civil registration number in part of the study period. For the remaining cases we retrieved medical records from the OUH.

\section{Case Definition}

Medical records of all potential cases were evaluated by one of us (D.G.), a neurology consultant with a special interest in myas- thenia. A diagnosis of myasthenia was considered definite if all 3 of the following criteria were fulfilled: (i) the recorded history and examination were compatible with myasthenia (weakness and fatiguability), (ii) the result of at least 1 positive paraclinical test (antibody test results, repetitive nerve stimulation, single-fibre electromyogram or edrophonium test) was recorded, and (iii) alternative disorders, if suspected, were ruled out by appropriate tests. If a diagnosis of myasthenia was stated in the medical record, but could not be definitely confirmed or ruled out due to insufficient information on one or more of the aforementioned cri- 
Fig. 2. Potential cases of myasthenia $(n=$ 112) in a Danish county in 1993-2008 identified by 3 registers. Only individuals presenting more than 1 pyridostigmine prescription were included in the Prescription Register.

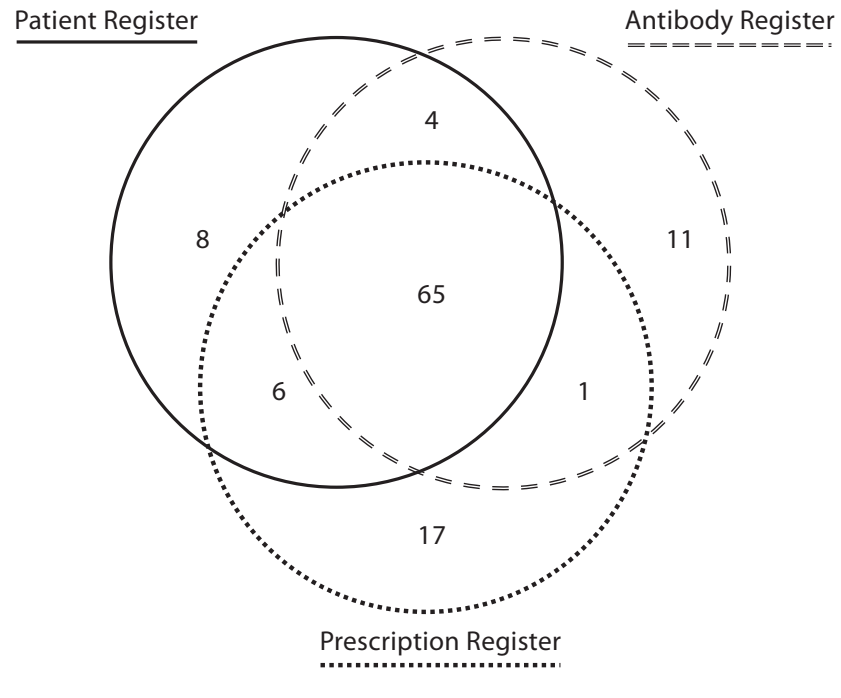

\section{Results}

was rejected if a case could not be classified as definite or possible. Cases with no identifiable medical records with pertinent information were classified as non-evaluable.

We also validated the diagnosis in two other samples identified exclusively from the Patient Register: one sample comprised all myasthenia coded contacts at any hospital in the Region of Southern Denmark (RSD; population 1.2 million; 4 neurology departments) and the other all myasthenia coded contacts to the Department of Neurology, RH (myasthenia referral centre in Eastern Denmark; population 2.45 million). We limited the material to potential incident cases of myasthenia as previously described. A random sample of 100 potential cases of myasthenia from each region was identified for further validation. The RSD also includes the County, and therefore there was some degree of overlap between the cases in this sample and those of the main study.

\section{Statistical Analysis}

Results were reported in numbers and percentages for categorical data and median and interquartile ranges for continuous variables. For group comparisons we used the $\chi^{2}$ test, Fisher's exact test and Mann-Whitney U test when appropriate. Probability results below 0.05 were considered statistically significant.

The positive predictive value of a register diagnosis (PPVD) with $95 \%$ confidence intervals was calculated as the proportion of individuals having myasthenia according to register data where the diagnosis could be verified according to our criteria.

The present study is part of a larger epidemiological research project that was approved by the Ethics Committee, RSD and the Danish Registry Board.

Use of Register Linkage to Identify

Patients with Myasthenia
In all, 193 potential cases of myasthenia (diagnosis code of myasthenia, presented prescription of pyridostigmine or positive antibody test) were identified in the County in the study period from 1993 to 2008 (fig. 1). Eighty-one individuals were excluded because they were registered before or after being eligible for the study. The distribution of the remaining 112 subjects among registers is presented in figure 2 .

The main analyses focused on the 101 potential incident cases of myasthenia recorded in the Patient or the Prescription Register (fig. 1). Age, gender and year of first registration were comparable in subjects identified by either the Patient Register $(n=83)$ or the Prescription Register $(\mathrm{n}=89$; table 1$)$. Subjects identified by the Prescription Register were less frequently seropositive (74.2\%) and had more frequently no registered antibody test (16.9\%) compared with subjects tracked by the Patient Register (83.1 and 2.4\%, respectively). We could not identify any hospital contacts (discharges or outpatient visits) coded under myasthenia for $18(20.2 \%)$ of the subjects identified in the Prescription Register.

Various indirect markers of validity were most frequent in the group of subjects identified in both registers. The percentage that tested seropositive (91.6\%) was highest and the percentage with no registered antibody test

Neuroepidemiology 2011;37:120-128 
Table 1. Characteristics of 101 potential incident myasthenia cases in a Danish county, 1993-2008

\begin{tabular}{|c|c|c|c|c|c|}
\hline & $\begin{array}{l}\text { Patient } \\
\text { Register } \\
(\mathrm{n}=83)\end{array}$ & $\begin{array}{l}\text { Prescription } \\
\text { Register } \\
(\mathrm{n}=89)\end{array}$ & $\begin{array}{l}\text { Patient and } \\
\text { Prescription } \\
\text { Register }(n=71)\end{array}$ & $\begin{array}{l}\text { Patient } \\
\text { Register } \\
\text { only }(n=12)\end{array}$ & $\begin{array}{l}\text { Prescription } \\
\text { Register } \\
\text { only }(n=18)\end{array}$ \\
\hline Female & $42(50.6)$ & $48(53.9)$ & $37(52.1)$ & $5(41.7)$ & $11(61.1)$ \\
\hline \multicolumn{6}{|l|}{ Age at first registration ${ }^{1}$} \\
\hline Median, years & $66.6[50.7-76.4]$ & $61.5[50.3-75.3]$ & $63.6[51.9-76.4]$ & $67.9[37.1-76.3]$ & $51.6[43.0-60.5]$ \\
\hline$<40$ years & $17(20.5)$ & $17(19.1)$ & $14(19.7)$ & $3(25.0)$ & $3(16.7)$ \\
\hline$\geq 40$ years & $66(79.5)$ & $72(80.9)$ & $57(80.3)$ & $9(75.0)$ & $15(83.3)$ \\
\hline \multicolumn{6}{|l|}{ Year of first registration } \\
\hline $1993-2000$ & $37(44.6)$ & $42(47.2)$ & $32(45.1)$ & $5(41.7)$ & $10(55.6)$ \\
\hline 2001-2008 & $46(55.4)$ & $47(52.8)$ & $39(54.9)$ & $7(58.3)$ & $8(44.4)$ \\
\hline \multicolumn{6}{|l|}{$A C h R a b^{2}$} \\
\hline Positive & $69(83.1)$ & $66(74.2)$ & $65(91.6)$ & $4(33.3)$ & $1(5.6)$ \\
\hline Negative & $12(14.5)$ & $8(9.0)$ & $5(7.0)$ & $7(58.3)$ & $3(16.7)$ \\
\hline Not registered & $2(2.4)$ & $15(16.9)$ & $1(1.4)$ & $1(8.3)$ & $14(77.8)$ \\
\hline \multicolumn{6}{|c|}{ Registered with diagnosis of myasthenia - most specialized department type ever ${ }^{3}$} \\
\hline Neurology & $78(94.0)$ & $69(77.5)$ & $69(97.2)$ & $9(75.0)$ & n.a. \\
\hline Non-neurology only & $5(6.0)$ & $2(2.3)$ & $2(2.8)$ & $3(25.0)$ & n.a. \\
\hline Not registered at any department & n.a. & $18(20.2)$ & n.a. & n.a. & n.a. \\
\hline \multicolumn{6}{|l|}{ Diagnosis verified by medical records } \\
\hline \multicolumn{6}{|l|}{ Yes } \\
\hline Definite & $71(85.5)$ & $66(74.2)$ & $66(93.0)$ & $5(41.7)$ & 0 \\
\hline Possible & $3(3.6)$ & $7(7.9)$ & $3(4.2)$ & 0 & $4(22.2)$ \\
\hline No & $9(10.8)$ & $9(10.1)$ & $2(2.8)$ & $7(58.3)$ & $7(38.9)$ \\
\hline Non-evaluable & 0 & $7(7.9)$ & 0 & 0 & $7(38.9)$ \\
\hline
\end{tabular}

Results are numbers with percentages in parentheses or medians with interquartile ranges in square brackets. n.a. = Not applicable.

${ }^{1}$ Age on date of the earliest of the following: registered myasthenia diagnosis, second presented pyridostigmine prescription or positive antibody test.
${ }^{2}$ According to the Antibody Register.

${ }^{3}$ The 'most specialized department' subject was seen at in- or outpatient examination and recorded with a primary code of myasthenia according to the Patient Register.

Table 2. Reasons for rejection of register myasthenia diagnosis in the 16 false-positive cases

$\begin{array}{lll}\begin{array}{l}\text { Patient and Pre- } \\ \text { scription }(\mathrm{n}=2)\end{array} & \begin{array}{l}\text { Patient only } \\ (\mathrm{n}=7)\end{array} & \begin{array}{l}\text { Prescription } \\ \text { only }(\mathrm{n}=7)\end{array} \\ 0 & 5 & 0 \\ 1 & 0 & 1 \\ 1 & 1 & 1 \\ 0 & \text { n.a. } & 5 \\ 0 & 1 & 0\end{array}$

Workup revealed that cause was other neurological disorder ${ }^{1}$ Myasthenia diagnosis initially suspected, but later rejected Diffuse symptomatology

Pyridostigmine used for indication other than myasthenia ${ }^{2}$ Coding error

\footnotetext{
n.a. = Not applicable.

${ }^{1}$ Includes the following: acute disseminated encephalomyelitis, brain stem infarction, cranial nerve affection (III or IV), Horner's syndrome.

${ }^{2}$ Given as symptomatic treatment in patients suffering from multiple sclerosis $(n=4)$ or subileus $(n=1)$.
} 
was lowest in this group (1.4\%) (antibody test vs. groups, $\mathrm{p}<0.001)$. The Prescription Register only group had the highest frequency of no registered antibody test $(77.8 \%$; table 1).

We could verify a diagnosis of definite myasthenia according to our criteria in $85.5 \%$ of patients identified in the Patient Register and $74.2 \%$ of those identified in the Prescription Register (table 1). The diagnosis could be verified as definite in $93.0 \%$ of subjects recorded in both registers, but only in 5 of 12 (41.7\%) of Patient Register only and none of 18 of Prescription Register only subjects ( $\mathrm{p}<0.001)$. In the Prescription only group, however, 7 cases $(38.9 \%)$ were non-evaluable.

We attempted to validate the diagnosis through medical records for (i) all 101 subjects included in the main analyses, (ii) for subjects only recorded in the Antibody Register ( $n=11$ ) and (iii) for two categories excluded by our register criteria, i.e. subjects only recorded in the $\mathrm{Pa}$ tient Register under secondary myasthenia codes $(n=7)$ and subjects only recorded in the Prescription Register with a single prescription of pyridostigmine $(n=33)$. In most groups the number of non-evaluable subjects was small. However, subjects only identified by prescriptions were a notable exception, regardless of whether identified by 2 or more pyridostigmine prescriptions ( 7 of 18 in the Prescription Register only group) or only a single prescription (16 of 33).

We verified the diagnosis of myasthenia in 85 subjects (75 definite and 10 possible cases). These figures include 3 definite and 1 possible case from the Antibody only group, 1 possible case with secondary myasthenia codes from the Patient Register only group, and 1 definite and 1 possible case identified among single pyridostigmine prescription presenters. In the Antibody Register only group, the 7 non-cases comprised 2 subjects who suffered from amyotrophic lateral sclerosis, 3 cases that, according to an evaluation by a neurologist with a special interest in myasthenia, did not have specific complaints or findings compatible with this disorder, and 2 non-evaluable cases.

In the 85 subjects with verified myasthenia diagnosis, the median age on the index date was 68.4 years (interquartile range 52.7-77.5). Forty-five of the verified cases were women $(52.4 \%), 72$ (84.7\%) were seropositive for AChRab, 13 (15\%) had ocular myasthenia, and 6 cases had histology-verified thymoma (7.1\%).

Information on the year of symptom onset was available in 78 of 85 subjects (91.7\%) with verified myasthenia. The median age at symptom onset was 66 years (interquartile range 52-76), and the majority of cases had their onset after the age of 40 (82.4\%). The female-to-male ratio was $4: 1$ in those aged 40 years or less and 0.8:1 in those aged 40 years or more at the time of symptom onset (sex vs. age in categories, $p=0.004$ ). In 42 of 78 verified cases (53.8\%), there was full agreement between the year of symptom onset and the year of the index date in the register. Allowing for a difference of 1 year between symptom onset and year of index date increased agreement to $83.3 \%$ ( $89.7 \%$ for 2 years of difference). The corresponding figures for the subsample of 68 verified cases from the Patient and Prescription Register group were 57.5, 81.8 and $87.9 \%$, respectively.

Reasons for rejecting the register diagnosis in falsepositive cases are presented by group in table 2 .

\section{PPVD in County Data}

The PPVD when cases were tracked using the Patient Register as the single source was $85.5 \%$ (95\% confidence interval, CI, 76.1-92.3) if only definite cases were included and $89.2 \%(80.4-94.9)$ if all cases (definite and possible) were included. For the Prescription Register the PPVD was $72.5 \%$ (95\% CI 62.2-81.4) for definite cases and $80.2 \%$ (70.6-87.8) for all cases. The PPVD of a diagnosis using the Antibody Register as a single register source is presented in table 3 .

The PPVD for patients identified in both the Patient Register and the Prescription Register were 92.9\% (95\% CI 84.3-97.7) for definite and 97.2\% (90.2-99.7) for definite and possible cases. The combination of Patient and Prescription Register also performed well when compared with other combinations of register sources (table 3).

In the Patient and Prescription Register group we examined the time window necessary for a subject to be traced in both registers. With a time window of maximum 3 months between events in the two registers we captured $70.4 \%$ of the subjects in the Patient and Prescription group, which increased to $87.3 \%$ if a time window of maximum 6 months was employed. Restricting the analysis to verified cases of myasthenia had little influence on these results (71.0 and $86.7 \%$, respectively).

\section{PPVD of Patient Register - Other Samples}

For the sample at the Neurology Department, RH, we could retrieve medical records for 98 out of 100 sampled patients. A definite diagnosis was established in 78 cases (PPVD of 78\%; 95\% CI 68.6-85.7). If the 2 possible cases were also included, the PPVD was $80 \%$ (70.8-87.3). In the sample from RSD we could retrieve medical records for 
Table 3. Positive predictive value of register diagnosis of myasthenia by data source

\begin{tabular}{|c|c|c|c|c|c|c|}
\hline \multirow[t]{2}{*}{ Source for case capture } & \multirow{2}{*}{$\begin{array}{l}\text { Potential cases } \\
\text { captured } \\
\text { evaluable } / \text { total }\end{array}$} & \multicolumn{2}{|c|}{ Positive predictive value, $\%$} & \multirow{2}{*}{$\begin{array}{l}\text { False- } \\
\text { positive }\end{array}$} & \multicolumn{2}{|l|}{ Sensitivity ${ }^{1}, \%$} \\
\hline & & definite only & definite and possible & & definite only & definite and possible \\
\hline \multicolumn{7}{|l|}{ Single register } \\
\hline Patient & $83 / 83$ & $85.5[76.1-92.3]$ & 89.2 [80.4-94.9] & $9(10.8)$ & 94.7 [86.9-98.5] & 87.1 [78.0-93.3] \\
\hline Prescription $^{3}$ & $82 / 89$ & $72.5[62.2-81.4]$ & $80.2[70.6-87.8]$ & $9(10.1)$ & $88.0[78.3-94.4]$ & $85.9[76.6-92.5]$ \\
\hline Antibody ${ }^{4,5}$ & $79 / 81$ & $84.0[74.1-91.2]$ & $88.9[80.0-94.8]$ & $8(9.9)$ & 90.7 [81.7-96.2] & 84.7 [75.3-91.6] \\
\hline \multicolumn{7}{|l|}{ Multiple registers } \\
\hline Patient and Prescription & $71 / 71$ & 92.9 [84.3-97.7] & 97.2 [90.2-99.7] & $2(2.8)$ & $88.0[78.4-94.4]$ & $81.2[71.2-88.8]$ \\
\hline Prescription and Antibody ${ }^{4}$ & $66 / 66$ & $93.9[85.2-98.3]$ & 98.5 [91.8-99.9] & $1(1.5)$ & $82.7[72.2-90.4]$ & $76.5[66.0-85.0]$ \\
\hline Patient and Antibody ${ }^{4}$ & $69 / 69$ & $94.2[85.8-98.4]$ & $97.1[89.9-99.6]$ & $2(2.9)$ & $86.7[76.8-93.4]$ & $78.8[68.6-86.9]$ \\
\hline $\begin{array}{l}\text { Patient and Prescription } \\
\text { and Antibody }{ }^{4}\end{array}$ & $64 / 64$ & 96.9 [89.2-99.6] & $100.0[94.4-100.0]$ & 0 & $82.7[72.2-90.4]$ & $75.3[64.7-84.0]$ \\
\hline $\begin{array}{l}\text { Results are numbers witl } \\
\text { cent with } 95 \% \text { CI in square } \\
{ }^{1} \text { Percentage of all defini } \\
\text { ( } \mathrm{n}=85 \text { ) verified cases in the } \\
\text { sible cases identified by the } \\
\text { ister only (secondary myasth } \\
\text { only (1 pyridostigmine presc }\end{array}$ & $\begin{array}{l}\text { percentages in } \\
\text { rackets. } \\
\text { ( }(n=75) \text { and } \\
\text { study; includes } \\
\text { Antibody Regist } \\
\text { enia code) and } \\
\text { ription only). }\end{array}$ & $\begin{array}{l}\text { parentheses or } \mathrm{p} \\
\text { definite and possi } \\
4 \text { definite and } 3 \mathrm{p} \\
\text { ter only, Patient } \mathrm{R} \\
\text { Prescription Regis }\end{array}$ & $\begin{array}{l}{ }^{2} \text { Medical r } \\
\text { myasthenia ca } \\
{ }^{3} \text { Only subj } \\
\text { tions in study } \\
{ }^{4} \text { Only sub } \\
{ }^{5} 11 \text { subjec } \\
\text { AChR only in }\end{array}$ & $\begin{array}{l}\text { cords with } \\
\text { es could b } \\
\text { cts presen } \\
\text { eriod incl } \\
\text { cts with p } \\
\text { s exclusive } \\
\text { uded in th }\end{array}$ & $\begin{array}{l}\text { nformation pertin } \\
\text { etrieved. } \\
\text { g } 2 \text { or more pyrid } \\
\text { ed. } \\
\text { itive antibody test } \\
\text { identified by pos } \\
\text { analysis. }\end{array}$ & $\begin{array}{l}\text { ent to evaluation of } \\
\text { stigmine prescrip- } \\
\text { included. } \\
\text { itive antibodies for }\end{array}$ \\
\hline
\end{tabular}

94 of 100 selected patients and reached a diagnosis of definite in 65 cases (PPV 65\%; 95\% 54.8-74.3) and definite or possible in 73 cases (PPVD 73\%; 63.2-81.4).

\section{Discussion}

We found that identifying patients with myasthenia by linkage of a number of population-based Danish registers was feasible. Combining Patient Register and Prescription Register information resulted in a high positive predictive value of the register diagnosis and acceptable sensitivity. We will apply the method in future populationbased epidemiological studies of myasthenia in Denmark.

We expect use of automated registers in myasthenia research to burgeon in years to come since many of these resources have now been in use sufficiently long to enable identification and follow-up of large samples of myasthenia patients at relatively low cost. Probably owing to logistical and data confidentiality issues, validation of the register diagnoses is frequently lacking in these studies [2-5]. Although differences in design make straightforward comparisons across studies difficult, we do believe that our study provides an impression of the validity of approaches used by other researchers, provided the settings are comparable to ours.

The Danish health system is highly organized with free access to health care. Most drugs, including pyridostigmine, are subsidized by the state. Combined with an extensive recording of health-related issues in a large variety of registers, the setting has highly desirable qualities for large-scale epidemiological studies. The register data are covered in an automated fashion making issues of recall bias irrelevant. The main issues to consider are the validity of the register diagnosis, here estimated by the PPVD, and the completeness of registration, i.e. the proportion of patients with myasthenia in the target population correctly classified as such by the register [14], here estimated by sensitivity.

The validity of a Patient Register diagnosis of myasthenia in the County was comparable to that of $\mathrm{RH}$ (PPVD 89.2 vs. 80.0\%). The lower PPVD detected in the sample from the RSD (73\%), might, at least in part, be due to our inability to retrieve medical records on 6 cases (6\%), 3 of which were registered with positive AChRab in the Antibody Register. Medical records were mainly retrieved from 5 neurology departments out of a total of 20 neurology departments in Denmark, which included 2 of 
the 4 university hospital departments that act as referral centres for myasthenia. We therefore believe our results to be generalizable to Denmark as a whole.

It is our experience that the vast majority of patients with myasthenia are referred to departments of neurology for further workup, and that only management of uncomplicated myasthenia after full hospital evaluation is on rare occasions handed over to privately practising neurologists or general practitioners. Patients with undiagnosed myasthenia would not be captured by our method. However, we assume this scenario to be rare, especially given the long study period. We therefore believe that our calculations of sensitivity presented in table 2 provide a reasonable estimate of completeness for the $\mathrm{Pa}$ tient Register.

Our prescription data contain no information on the indication for pyridostigmine use. This was particularly problematic in subjects identified exclusively through the Prescription Register. We could evaluate the indication for pyridostigmine use through medical records for a small number of patients from this group. Based on this, we suspect that the bulk of cases only recorded in the Prescription Register represented use of pyridostigmine for indications other than myasthenia, or an initial loosely founded suspicion of myasthenia in patients where the diagnosis was later rejected.

We found that combining Patient and Prescription Register data to a large degree overcame issues of validity in the County sample. There is an inherent survival bias when using any of the sources in combination, as opposed to using a single source. To be recorded in the $\mathrm{Pa}$ tient and Prescription Register for instance, the patient has to live long enough to be seen at the hospital and present a prescription. It was therefore reassuring that these events were separated by a time window of less than 6 months in $87.3 \%$ of cases captured in the Patient and Prescription Register group. Severe myasthenia leading to death shortly after presentation is extremely rare.

The characteristics of verified myasthenia cases in the County sample were highly comparable to those reported in previous epidemiological studies with regard to age and gender distribution, in particular female-to-male ratio within age categories, and myasthenia subtype (ocular vs. generalized) [1, 15]. A small minority of myasthenia patients, previously classified as antibody negative, have been shown to have antibodies against a kinase (MUSK). In our experience, the incidence of MUSK-positive myasthenia is very low in Denmark. Therefore, lack of this information in this or future register-based Danish studies only represents a minor problem.

Use of Register Linkage to Identify

Patients with Myasthenia
Identifying incident cases of myasthenia is, regardless of method, hampered by the lag time between symptom onset and recognition of this rare disorder. We therefore find it acceptable that the year of symptom onset coincided with the year of index date in roughly half the cases and that the majority of remaining cases were recorded within a couple of years of symptom onset. This finding did not depend on the number or combination of registers used for case identification, which underscores that the problem is not inherent to the method suggested here of linking Patient and Prescription Register data.

Patient Register data are routinely collected and made available for research at a nationwide level in Denmark. A national prescription database with a data structure and content highly reminiscent of the County Prescription Register has recorded all prescriptions presented in Denmark since 1995 [9]. We believe this set-up provides unique opportunities for research in myasthenia that our method will enable us to utilize. Similar nationwide registers may provide the basis for use of this method in other countries with health systems comparable to the Danish one.

\section{Acknowledgments}

We wish to thank other departments in the RSD for retrieving medical records, in particular the departments of Neurology in Esbjerg, Sønderborg and Vejle.

\section{Disclosure Statement}

The study received support from the Savværkejer Jeppe Juhl og hustru Ovita Juhls Mindelegat fund. No conflict of interest.

References

Neuroepidemiology 2011;37:120-128 
5 Andersen JB, Engeland A, Owe JF, et al: Myasthenia gravis requiring pyridostigmine treatment in a national population cohort. Eur J Neurol 2010;17:1445-1450.

6 Christensen PB, Jensen TS, Tsiropoulos I, et al: Incidence and prevalence of myasthenia gravis in western Denmark: 1975 to 1989. Neurology 1993;43:1779-1783.

77 Frank L: When an entire country is a cohort. Science 2000;287:2398-2399.

$\checkmark 8$ Andersen TF, Madsen M, Jørgensen J, et al: The Danish National Hospital Register: a national source of data for modern health sciences. Dan Med Bull 1999;46:263-268.
9 Gaist D, Sørensen HT, Hallas J: The Danish prescription registries. Dan Med Bull 1997; 44:445-448.

10 WHO Collaborating Centre for Drug Statistics Methodology: Anatomical Therapeutic Chemical (ATC) Classification Index with Defined Daily Doses (DDDs). Oslo, WHO, 2000.

11 Gammeltoft S, Somnier FE: Acetylcholine receptor antibodies in myasthenia gravis: measurements of plasma concentrations by immunoprecipitation. Ugeskr Læger 1984; 146:352-355.

12 Kennel PF, Vilquin J-T, Braun S, et al: Myasthenia gravis: comparative autoantibody assays using human muscle, TE671 and glucocorticoid-treated TE671 cells as sources of antigen. Clin Immunol Immunopathol 1995;74:293-296.
13 Pedersen CB, Gøtzsche H, Møller JØ, et al: The Danish civil registration system - a cohort of 8 million people. Dan Med Bull 2006; 53:441-449.

14 Sørensen HT, Sabroe S, Olsen J: A framework for evaluation of secondary data sources in epidemiological research. Intern J Epidemiol 1996;25:435-442.

15 Grob D, Brunner N, Namba T: Lifetime course of myasthenia gravis. Muscle Nerve 2008;37:141-149. 